\title{
A good stretch regulates satiety
}

The regulation of energy balance involves the integration of food intake-related signals from

food-intakeinduced intestinal stretch activates mechanoreceptors in the intestinal wall to generate satiety signals

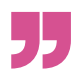
underlying peripheral molecular and cellular signalling pathways are poorly understood. Now, Bai et al. show that food-intakewall to generate satiety signals. cell bodies in the nodose ganglia the periphery by the brain, but the induced intestinal stretch activates mechanoreceptors in the intestinal The vagus nerve is a key conduit of interoceptive information from the periphery. Vagal afferents have their and their axons bifurcate into two branches, with one terminating in the brainstem in areas such as the nucleus tractus solitarius (NTS) and the other terminating in the gut. These latter terminals can form several different types of ending, including 'mucosal' endings that are specialized for the detection of chemical signals (for example, hormones, $\mathrm{pH}$ or nutrients) and intraganglionic laminar endings (IGLEs) that detect mechanical stimuli such as gastrointestinal tract (GIT)

distension. Vagal afferents are therefore in a position to play a part in the regulation of food intake and satiety by integrating chemical and mechanical stimuli.

In the new study, the authors first used single-cell RNA sequencing (scRNAseq) and viral tracing methods to generate a transcriptomic map of vagal afferent subtypes in order to identify molecular markers that are specific to these subtypes. They then used this information to map, target and manipulate the different subtypes individually and to determine their specific roles in food intake behaviour.

The transcriptomic data revealed 12 sets of genetically distinct GIT-innervating vagal sensory neurons, and for most of these groups, a unique genetic marker could be identified. In general, different regions of the GIT were innervated by different vagal sensory afferents: for example, somatostatin (Sst) and calcitonin-related polypeptide (Calca) each labelled approximately $10 \%$ of stomach-projecting afferents. These findings were confirmed and extended by using Cre mouse lines combined with adeno-associated virus-mediated induction of a tdTomato reporter to visualize the gut innervation patterns of the various sensory afferent subtypes. This revealed that $S s t^{\mathrm{CRE}}$ and $\mathrm{Calca}^{\mathrm{CRE}}$ neurons formed mucosal endings in non-overlapping parts of the stomach, whereas vasointestinal peptide-Cre $\left(V i p^{\mathrm{CRE}}\right)$ mice showed expression of this mucosal type exclusively in the intestine. The authors also showed that there are two distinct populations of mechanosensitive afferents that form IGLEs, Glp1R neurons that innervate the stomach and Oxtr neurons that innervate the intestines.
To investigate functional roles, the authors expressed the excitatory opsin channelrhodopsin 2 (ChR2) in two sets of mucosal-ending neurons (Vip ${ }^{\mathrm{CRE}}$ and Gpr65 ${ }^{\mathrm{CRE}}$ ) and two mechanoreceptors (Oxtr ${ }^{\mathrm{CRE}}$ and $G l p 1 r^{\mathrm{CRE}}$ ). Optical stimulation in the NTS of mucosal neuron terminals increased FOS expression in their downstream targets (indicating activation) but had no effect on food intake. By contrast, optical stimulation of IGLE terminals resulted in a substantial reduction in food intake, suggesting that $O x t r$ and Glp1r vagal afferents contribute to the regulation of food intake.

Most previous studies on the role of stretch in the regulation of food intake have focused on the stomach rather than the intestines. To test the ability of stomach and intestinal stretch to inhibit hypothalamic agouti-related peptide (AgRP) neurons and regulate food intake independently of nutrient content, the authors developed multiple nonnutritive solutions that increased fluid (and thus mechanical stretch) in either the stomach or the intestine. Solutions that induced intestinal stretch, but not gastric stretch, reduced food intake and AgRP neuronal activity, whereas solutions that induced gastric stretch alone did not affect either food intake or AgRP neuronal activity. Infusion of non-nutritive solutions directly into the intestines (thereby bypassing the stomach) also inhibited both food intake and AgRP neurons.

Together, these findings suggest that mechanical intestinal stretch is crucial for the regulation of satiety following food ingestion.

Sian Lewis

ORIGINAL ARTICLE Bai, L. et al. Genetic identification of vagal sensory neurons that control feeding. Cell 179, 1129-1143 (2019) 